\title{
Bearing Capacity Analysis of Spherical Hybrid Gas Bearings with Tangential Gas Supply
}

\author{
Chenhui Jia ${ }^{1, a}$, Jing Gao ${ }^{1, b^{*}}$, Ming Qiu ${ }^{1, c}$ and Wensuo Ma ${ }^{1, d}$ \\ ${ }^{1}$ School of Mechatronics Engineering, Henan University of Science and Technology, Luoyang \\ 471003, China. \\ axjiachenhui@163.com, b15838842452@163.com, \\ 'qiuming69@126.com, ${ }^{\mathrm{d}}$ mawensuo@126.com
}

\begin{abstract}
In order to research the static performances of the spherical spiral groove hybrid gas bearings, the lubrication analysis mathematical model of spherical hybrid gas bearings is established with variable tangential angels of gas supply. Subsequently, the mathematical model is transformed by derivatives and integrals methods and finite difference method. Homogeneous coordinate system of hybrid gas bearings is established in this process, and transformation with angle retained and oblique coordinate transformation are employed to the mathematical model. The difference expressions of gas film stable pressure control equations are established. The method of dividing grids is employed to the solution region, so the stable pressure distribution of gas film is numerically calculated by using $\mathrm{VC}++6.0$ software. The bearing capacity is obtained by integration of gas film circumferential and radial pressure. The influence law of supply pressure and supply tangential angle on bearing capacity is researched in different eccentricity and speed. The results show that parameters of gas bearings need to be chosen properly to get better bearing capacity.
\end{abstract}

Keywords: Lubrication analysis mathematical model; Finite difference method; Hybrid gas bearings; Tangential gas supply; Bearing capacity.

\section{切向供气球面动静压气体轴承承载力分析}

\author{
贾晨辉 ${ }^{1}$ ，高靖 ${ }^{1}$ ，邱明 ${ }^{1}$ ，马文锁 ${ }^{1}$
}

(1 河南科技大学机电工程学院, 河南洛阳471003)

摘要: 以半球面螺旋槽气体动静压轴承为研究对象, 建立供气切向角可变的球面动静压气体轴承润滑分析数学模型。在 广义坐标系下对数学模型进行保角变换和斜坐标变换, 结合导数积分法与有限差分法, 建立气膜稳态压力控制方程的差分表 达式, 进行求解域网格划分, 并采用 $V C++6.0$ 编程数值计算稳态气膜压力分布; 利用辛普森公式对气膜周向与径向压力进 行积分, 求得气体轴承的稳态承载力。在不同偏心率、转速条件下, 研究不同轴承参数 (供气压力、供气切向角度) 对轴承 稳态承载力的影响规律, 结果表明选择合适的轴承参数可提高承载力。

关键词: 润滑分析数学模型; 有限差分法; 动静压气体轴承; 切向供气；承载力

中图分类号: TM 344.1 文献标志码：A

引言

随着科学技术的发展, 高转速机械已成为高效率生产的工具, 在许多应用领域, 气体轴承可以提高机 械的工作效率。气体轴承支撑的转子转速高, 无噪声、摩擦小显现许多传统轴承没有的优点 [1]。在微细 工程、医疗机械、空间技术等领域中得到极大的应用。

采用小孔节流的球面螺旋槽动静压气体轴承是一种新型结构的气体轴承。它综合了静压与动压轴承的 优点, 即在转子启停阶段使用静压气体轴承的供气方式, 形成静压承载能力, 避免了干摩擦; 当转子高速 旋转时, 利用螺旋槽产生强的动压效应形成动压承载能力, 避免了支撑轴承所需的持续高压供气 [3], 较 
同类轴承有较高的承载力。

本文采用有限差分法 [5] 求解数学模型, 通过编程计算得到气膜的稳态压力分布, 求出气膜的稳态承 载力。研究不同轴承参数 (供气压力、供气切向角度) 对轴承稳态承载力的影响规律。

\section{1 球面螺旋槽动静压气体轴承结构}

图 1 为球面螺旋槽动静压气体轴承的结构图, 可以看出轴承的周向方向分布两排节流孔。 $\beta$ 是螺旋角; $\omega$ 是轴颈转速; $\varphi$ 是供气切向角; $\alpha 0$ 是转子小端角度; $\alpha 1$ 是螺旋槽起始端角度; $\alpha 2$ 是转子大端角度; ps 是小孔供气压力; br 是台宽; bg 是槽宽; hg 是槽区间隙。 h0 是台域内气膜平均间隙。

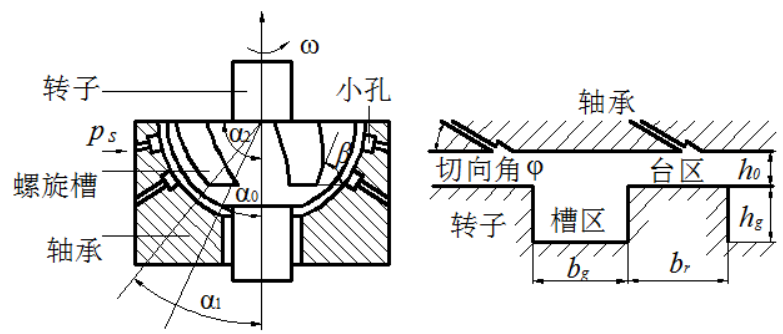

图 1 球面螺旋槽动静压气体轴承剖面示意图

\section{2 球面螺旋槽动静压气体轴承润滑分析数学模型}

以气体润滑运动方程、连续性方程、状态方程以及节流孔流量方程为基础，结合牛顿粘性定律和雷诺 方程假设, 在球坐标系下, 推导供气切向角可变的球面动静压气体轴承非线性无量纲雷诺方程 [6]。

$$
\begin{aligned}
& \sin \alpha \frac{\partial}{\partial \alpha}\left(\sin \alpha H^{3}\left(\frac{\partial P^{2}}{\partial \alpha}\right)\right)+\frac{\partial}{\partial \phi}\left(H^{3}\left(\frac{\partial P^{2}}{\partial \phi}\right)\right)+\delta_{k}\left(Q \sin \varphi+\frac{Q h_{0}}{2 R_{0} \sin \alpha} \cos \varphi \frac{\partial H}{\partial \phi}\right) \\
& =2 \Lambda \sin ^{2} \alpha \frac{\partial}{\partial \phi}(P H)+2 \sigma \sin ^{2} \alpha \frac{\partial}{\partial T}(P H)
\end{aligned}
$$

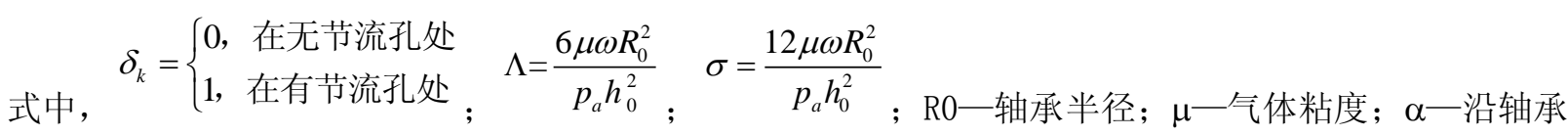
子午线方向的坐标; $\phi$ 一沿轴承周向方向的坐标; $\mathrm{pa}$ 一标准大气压强; Q一节流孔引入的气体质量流量因子。

$$
Q=\frac{24 \mu R_{0}^{2} \sin ^{2} \alpha}{\rho_{a} p_{a} h_{0}^{3}} \rho \tilde{v}
$$

式 (1) 所示的雷诺方程过于复杂, 无法直接求解, 为此须对其进行保角变化。变换形式取 $\xi=\ln (\tan (\alpha / 2))$, 带入 (1) 后即可将其变换成求解域为标准矩形的球面动静压气体润滑方程。当 $\alpha=0$ 时 保角变换不存在，对于本文研究的气体轴承不存在 $\alpha=0$ 的情况，故可直接变换 [10]。将式（1）变形为：

$$
\begin{aligned}
& \frac{\partial}{\partial \xi}\left(H^{3}\left(\frac{\partial P^{2}}{\partial \xi}\right)\right)+\frac{\partial}{\partial \phi}\left(H^{3}\left(\frac{\partial P^{2}}{\partial \phi}\right)\right)+\delta_{k}\left(Q \sin \varphi+\frac{Q h_{0}}{2 R_{0} \sin \alpha} \cos \varphi \frac{\partial H}{\partial \phi}\right) \\
& =2 \Lambda \gamma \frac{\partial}{\partial \phi}(P H)+2 \sigma \gamma \frac{\partial}{\partial T}(P H)
\end{aligned}
$$

式中, $\gamma=\sin ^{2}\left(2 \arctan e^{\xi}\right)$ 


\section{3 轴承稳态承载力的求解}

\section{1 数值计算稳态压力}

由于轴承的稳态雷诺方程与时间项无关, 故球面动静压气体轴承的稳态润滑分析无量纲雷诺方程:

$$
\begin{aligned}
& \frac{\partial}{\partial \xi}\left(H^{3}\left(\frac{\partial P^{2}}{\partial \xi}\right)\right)+\frac{\partial}{\partial \phi}\left(H^{3}\left(\frac{\partial P^{2}}{\partial \phi}\right)\right)+\delta_{k}\left(Q \sin \varphi+\frac{Q h_{0}}{2 R_{0} \sin \alpha} \cos \varphi \frac{\partial H}{\partial \phi}\right) \\
& =2 \Lambda \gamma \frac{\partial}{\partial \phi}(P H)
\end{aligned}
$$

在广义坐标系下, 采用有限差分法对式 (4) 离散化, 推导出稳态压力的差分表达式。为了计算的方 便和精确, 在斜坐标系下 $(x=\phi+\xi / \tan \beta, y=-\xi / \sin \beta)$ 划分网格, 划分网格时网格线要划分在槽台交界线上 [11], 如图 2 所示。由于求解域内气膜厚度是不连续的, 在求解雷诺方程时分为连续 (包括小孔区域)、 不连续两种情况。

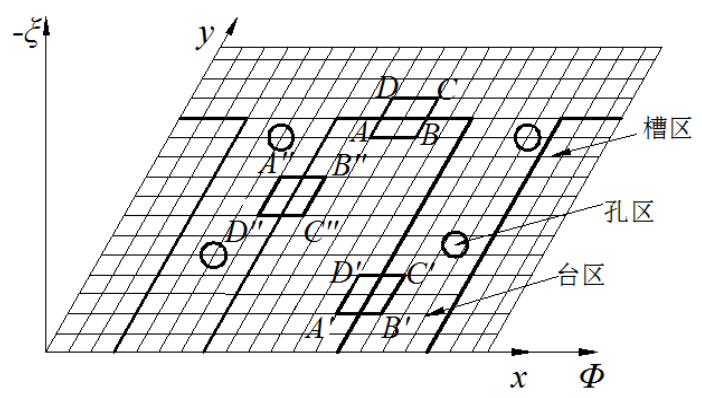

图 2 求解域网格划分示意图

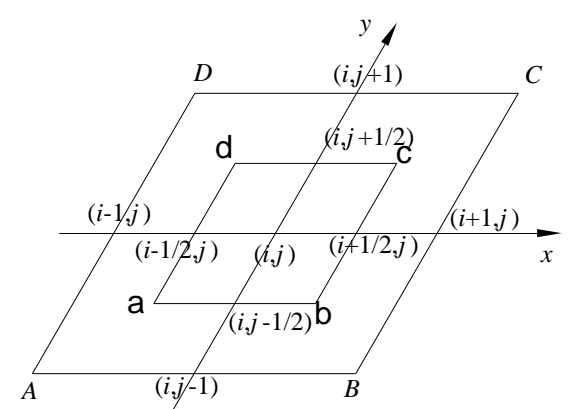

图 3 气膜厚度连续区域的网格

以求解小孔区域为例, 对式 (4) 等号两侧在每个网格节点的平行四边形求解域 abcd（记作 $D_{i, j}$ 如图 3 所示）上进行面积分得：

$$
\begin{aligned}
& \iint_{D_{i, j}}\left[\frac{\partial}{\partial \xi}\left(H^{3}\left(\frac{\partial P^{2}}{\partial \xi}\right)\right)+\frac{\partial}{\partial \phi}\left(H^{3}\left(\frac{\partial P^{2}}{\partial \phi}\right)\right)\right] d \phi d \xi=\iint_{D_{i, j}} 2 \Lambda \gamma \frac{\partial}{\partial \phi}(P H) d \phi d \xi \\
& -\iint_{D_{i, j}}\left(\frac{h_{0} Q \cos \varphi}{2 R_{0} \sin \alpha} \frac{\partial H}{\partial \phi}\right) d \phi d \xi-\iint_{D_{i, j}} Q \sin \varphi d \phi d \xi
\end{aligned}
$$

再利用格林公式将上式转化为相应区域 $C_{i, j}$ 的线积分:

$$
\begin{aligned}
& \int_{C_{i, j}} H^{3} \frac{\partial P^{2}}{\partial \phi} d \xi-\int_{C_{i, j}} H^{3} \frac{\partial P^{2}}{\partial \xi} d \phi=2 \Lambda \gamma \int_{C_{i, j}} P H d \xi-\frac{h_{0} Q \cos \varphi}{2 R_{0} \sin \alpha} \int_{C_{i, j}} H d \xi \\
& -Q \sin \varphi(\Delta x+\Delta y \cos \beta) \Delta y(-\sin \beta)
\end{aligned}
$$


(1)

采用有限差分法对式（6）离散化处理得到气膜连续域内控制方程在斜坐标系下的差分表达式:

$$
A_{i, j} P_{i, j}^{2}+B_{i, j} P_{i, j}+C_{i, j}=0
$$

气膜厚度不连续区域差分表达式的求解方法与连续区域差分表达式的求解方法相同。

求解稳态雷诺方程（4）采用如下边界条件：

大气边界条件：

$$
P\left(\alpha_{2}\right)=P\left(\alpha_{1}\right)=0
$$

对称边界条件：

$$
\left.\frac{\partial P}{\partial \phi}\right|_{\phi}=\left.\frac{\partial P}{\partial \phi}\right|_{\phi+2 \pi}
$$

节流孔边界条件:

$$
P=P_{d}
$$

式中， $P_{d}$ 一节流孔后端压力。

(2)

采用超松弛迭代法对式 (7) 求解, 得 $P_{i, j}$ 的表达式为

$P_{i, j}^{k}=P_{i, j}^{k-1}-\lambda\left[P_{i, j}^{k-1}-\left|\frac{\sqrt{B_{i, j}^{2}-4 A_{i, j} C_{i, j}}-B_{i, j}}{2 A_{i, j}}\right|\right]$

(3)

式中, $\lambda$ 一松弛因子, 一般可在 $1^{\sim} 2$ 之间选取; $k$ 一迭代次数。

当迭代结果达到足够精度后，终止迭代。通常采用相对收玫准则为：

$$
\frac{\sum_{j=1}^{n-1} \sum_{i=0}^{m}\left|P_{i, j}^{(\mathrm{k})}-P_{i, j}^{(k-1)}\right|}{\sum_{j=1}^{n-1} \sum_{i=0}^{m}\left|P_{i, j}^{(\mathrm{k})}\right|} \leq \delta
$$

一般取 $\delta-0.0001$ 。

同理，可以求出气膜厚度不连续区域气膜的压力分布。

本文采用表 1 所示的轴承设计参数进行数值计算。

表 1 动静压气体轴承设计参数

\begin{tabular}{cc}
\hline 名称 & 数值 \\
\hline 轴承半径 $R_{0}(\mathrm{~mm})$ & 4.8
\end{tabular}




\begin{tabular}{cc}
\hline 气膜平均间隙 $h_{0}(\mathrm{~mm})$ & 0.0012 \\
节流孔直径 $d_{1}(\mathrm{~mm})$ & 0.2 \\
供气孔个数 $r$ & 6 \\
螺旋角 $\beta\left(^{\circ}\right)$ & 60 \\
槽数 $N g$ & 6 \\
槽深比 $H g$ & 2.5 \\
槽宽比 $B g$ & 0.4 \\
节流孔系数 & 0.8 \\
槽起始端角度 $\alpha_{1}\left({ }^{\circ}\right)$ & 47 \\
大气压强 $p_{a}(\mathrm{MPa})$ & 0.1 \\
气体密度 $\rho_{a}\left(\mathrm{Kg} / \mathrm{m}^{3}\right)$ & 1.204 \\
气体粘度 $\mu\left(\mathrm{N} \cdot \mathrm{s} / \mathrm{m}^{2}\right)$ & $1.82 \times 10^{-5}$ \\
绝热比 $k$ & 1.4 \\
转子小端角度 $\alpha_{0}\left(^{\circ}\right)$ & 25 \\
转子大端角度 $\alpha_{2}\left(^{\circ}\right)$ & 90 \\
径向偏心率 $\varepsilon$ & 0.3 \\
轴向偏心率 $\varepsilon_{1}$ & 0.1 \\
\hline & \\
\hline
\end{tabular}

\section{2 数值计算稳态承载力}

采用辛普森复合积分法, 对气膜周向和径向压力积分, 得到沿 $\mathrm{x}$ 方向的承载力 $F_{x}$ 、沿 $\mathrm{y}$ 方向的承载力 $F_{y}$ 以及沿轴向 $\mathrm{z}$ 的承载力 $F_{z}$ :

$$
\begin{aligned}
& F_{x}=p_{a} R_{0}^{2} \int_{\theta_{2}}^{\theta_{0}} \int_{0}^{2 \pi}(p-1) \sin \phi \sin ^{2} \alpha d \alpha d \phi \\
& F_{y}=p_{a} R_{0}^{2} \int_{\theta_{2}}^{\theta_{0}} \int_{0}^{2 \pi}(p-1) \cos \phi \sin ^{2} \alpha d \alpha d \phi \\
& F_{z}=p_{a} R_{0}^{2} \int_{\theta_{2}}^{\theta_{0}} \int_{0}^{2 \pi}(p-1) \cos \alpha \sin \alpha d \alpha d \phi
\end{aligned}
$$

将上式无量纲化得 $W_{x} 、 W_{y}$ 和 $W_{z}$, 采用辛普森积分法, 对其进行数值求解, 得到轴承承载力:

$$
F_{x}=p_{a} R_{0}^{2} W_{x}
$$

同理可以计算其它两个方向的承载力。

\section{4 稳态承载力数值分析}

由图 4 可知, 随着供气压力的增加, 静压效应增强, 轴承的承载力增大; 随着转速的逐渐增加, 供气 压力的增大对轴承承载力增加的影响减弱。 


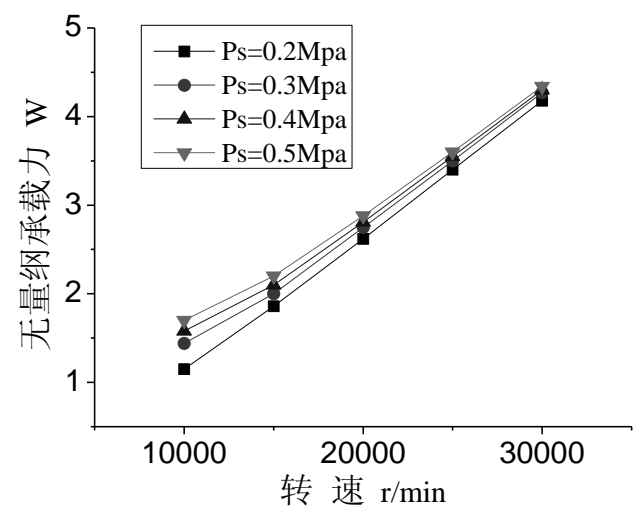

图 4 不同转速、供气压力对承载力的影响

由图 5 可知, 随着转速的增加, 轴承的承载力增大; 随着供气切向角度的增加, 轴承的承载力先减小 后增大; 转速越大, 供气切向角度对轴承承载力的影响变弱。

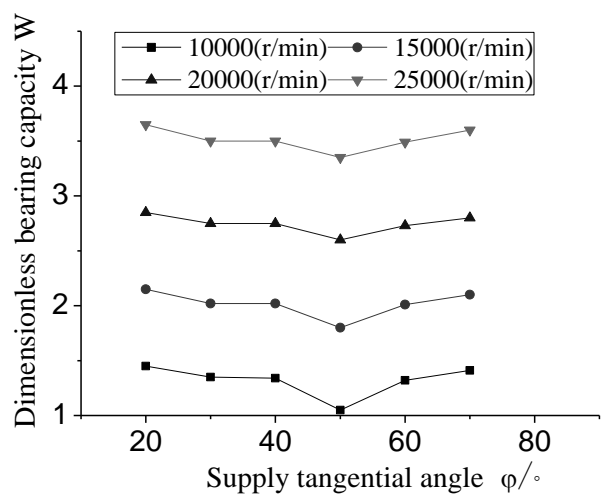

图 5 不同转速、供气切向角度对承载力的影响

\section{5 结论}

(1) 采用有限差分法求解控制方程, 有利于数值计算轴承的气膜压力分布。本文对供气切向角可变的 球面螺旋槽动静压气体轴承的求解方法, 同样适用于其他结构动静压气体轴承控制方程的求解。

(2) 通过分析轴承参数 (供气压力、供气切向角度) 对轴承稳态承载力的影响规律, 选择合适的轴承 设计参数, 可提高半球面螺旋槽气体动静压轴承的稳态承载性能。

\section{6 致谢}

本文由国家自然科学基金（51475142）；河南省科技创新杰出人才计划项目（154200510013）支持。

\section{Acknowledgement}

This work is supported by National Natural Science Foundation of China (Grant no: 51475142), and Project for Innovative outstanding person plan (in Science and Technology) of Henan Province (Grant no: 154200510013).

\section{参考文献:}

[1] Czolczyr1ski K, Kapitaniak K, Marynowski K. Stability of rotors supported in gas bearings with bushes mounted in air rings[J]. Wear, 1996, 199(199): 100-112.

[2] 许怀锦, 刘占生, 吕伟剑等. 气体润滑弹性波箔片轴承动态特性分析 [J]. 航空动力学报, 2011, 26(5)： $1185-1193$. 
[3] 秦冬黎, 姚英学. 小孔节流动静压混合气体润滑球轴承的干扰力矩分析 $[J]$. 润滑与密封, 2007, 32(4): 131-135.

[4] 龚智辉，宾鸿赞. 螺旋角的广义定义及其应用 $[J]$. 中国机械工程，1994，（2）：14-15.

[5] 王学敏, 杜建军, 李姗姗. 基于有限差分法对不同润滑介质下静压气体轴颈轴承性能研究 [J]. 机械工程学报, 2012, 48(3): 121-127.

[6] 刘噋, 刘育华, 陈世杰. 静压气体润滑 [M]. 哈尔滨：哈尔滨工业大学出版社, 1990.

[7] 周恒, 刘延柱. 气体动压轴承的原理及计算 $[\mathrm{M}]$. 国防工业出版社, 1981.

[8] 张广辉. 高速动静压混合气体轴承转子系统动力学特性研究 [D]. 哈尔滨: 哈尔滨工业大学博士学位论文, 2010.

[9］李树森，孟庆金金，刘噋. 小孔节流静压气体轴颈轴承的静态特性研究 [J]. 润滑与密封，2006, (2)：20-21.

[10] Jun Liu, and Mochimaru Yoshihiro. Analysis of oil-1ubricated herringbone grooved journal bearing with trapezoidal cross-section, using a spectral finite difference method [J]. Journal of Hydrodynamics, 2010, $22(5)$ : $408-412$.

[11] 卢志伟, 刘波, 张君安. 圆雉形螺旋槽气体动压轴承的数值分析 $[J]$. 轴承, 2008, (8) : 28-32.

\section{References}

[1] Czolczyrlski K, Kapitaniak K, Marynowski K: "Stability of rotors supported in gas bearings with bushes mounted in air rings" [J]. Wear, Vol. 199(1996), No.199, p.100-112.

[2] Xu Huai-jin, Liu Zhan-sheng, Lu Wei-jian, et al: "Dynamic characteristics analysis of bump foils journal bearings with air lubrication" [J]. Journal of Aerospace Power, Vol. 26(2011), No.5, p.1185-1193. (in Chinese)

[3] Qin Dong-li, Yao Ying-xue: "Disturbing torque analysis of orifice compensated hybrid gas spherical bearing" [J]. Lubrication Engineering, Vol. 32(2007), No.4, p.131-135. (in Chinese)

[4] Gong Zhi-hui, Bin Hong-zan: "Generalized definition and application of spiral angle" [J]. Mechanical Engineering Journal, (1994), No.2, p.14-15. (in Chinese)

[5] Wang Xue-ming, Du Jian-jun, Li Shan-shan: "Performance research on the externally pressurized gas journal bearing under different working gas based on finite difference method" [J]. Journal of Mechanical Engineering, Vol. 48(2012), No.3, p.121-127. (in Chinese)

[6] Liu Dun, Liu Yu-hua, Chen Shi-jie: Static Pressure Gas Lubrication (Harbin Institute of Technology Press, China 1990).

[7] Zhou Heng, Liu Yan-zhu: The Principle and Calculation of Dynamic Gas Bearings (National Defence Industry Press, China 1981).

[8] Zhang Guang-hui: Research of Dynamic Characteristics for High Speed Hybrid Gas Bearing Rotor System (Ph.D., Harbin Institute of Technology, China 2010).

[9] Li Shu-sen, Meng Qing-xin, Liu Tun: "Research of static characteristics for externally pressurized gas journal bearing with orifice compensated" [J]. Lubrication Engineering, (2006), No.2, p.20-21. (in Chinese)

[10] Jun Liu, Mochimaru Yoshihiro: “Analysis of oil-lubricated herringbone grooved journal bearing with trapezoidal cross-section, using a spectral finite difference method" [J]. Journal of Hydrodynamics, Vol. 22(2010), No.5, p.408-412.

[11] Lu Zhi-wei, Liu Bo, Zhang Jun-an: "Numerical analysis on conical spiral groove aerodynamic bearing" [J]. Bearing, (2008), No.8, p.28-32. (in Chinese) 